\title{
Uncertainty of Temperature Measurement in Working Environment
}

\author{
Tatiana Kelemenová ${ }^{1}$, Michal Kelemen ${ }^{1, *}$ \\ 1 Technical University of Košice, Faculty of Mechanical Engineering, Letná 9, 04200 Košice
}

\begin{abstract}
The uncertainty of measurement is a key component of expressing of measurement result. Knowledge of uncertainty is needed for balance of the process reliability. Every measurement device is not perfect and producer has to declare the maximum accuracy of the device and this can be used as a part of uncertainty. Paper deals with uncertainty analysis of temperature process measurement inside the working environment.
\end{abstract}

Keywords: uncertainty of measurement, temperature, error, gauge, reliability.

\section{Introduction}

Measurement results are very often basic leading point for making of decision and these results often influence the other phenomena's and processes. This paper deals with expression of with uncertainty of measurement and also about the including of them into the engineering measurement process. If we want to express measurement result, it is necessary to obtain the selected quantitative indicators of quality of results. This evaluation shows the reliability of measurement results. Comparison of results can be executed only in case, if there is also available uncertainty of these measurement. This is a reason for existence of generally accepted process of determination of measurement uncertainty [1,2].

Almost all areas of science need experimental works with expressing of measurement results. Also production process has to be checked for the quality of process and measurement results are as the base for the decision making for intervention into the production process. Measurement uncertainty has direct impact to achieved production quality.

There are several national and international standards for evaluation of measurement uncertainty and calibration process [3-7].

\section{Measurement of temperature using the resistive sensors}

Temperature is very frequently measured quantity in industry. Various sensors are currently used for this measurement. These sensors change the any quantity as electrical resistance, volume, length, pressure and shape in accordance with temperature change. Consequently, the measurement of temperature is based on measurement of changes influenced by temperature. It is necessary to select suitable principle of sensor according to concrete application and expected range of measured temperature. Resistive sensors are one of the possible way for industrial conditions. As material are used platinum, nickel, cooper or semiconductors.

Platinum has excellent properties as large and time stabile temperature coefficient. It is usable in temperature range from $-190^{\circ} \mathrm{C}$ up to $+630^{\circ} \mathrm{C}$. There are available various versions of platinum resistive sensors for industrial application (Fig. 1). 

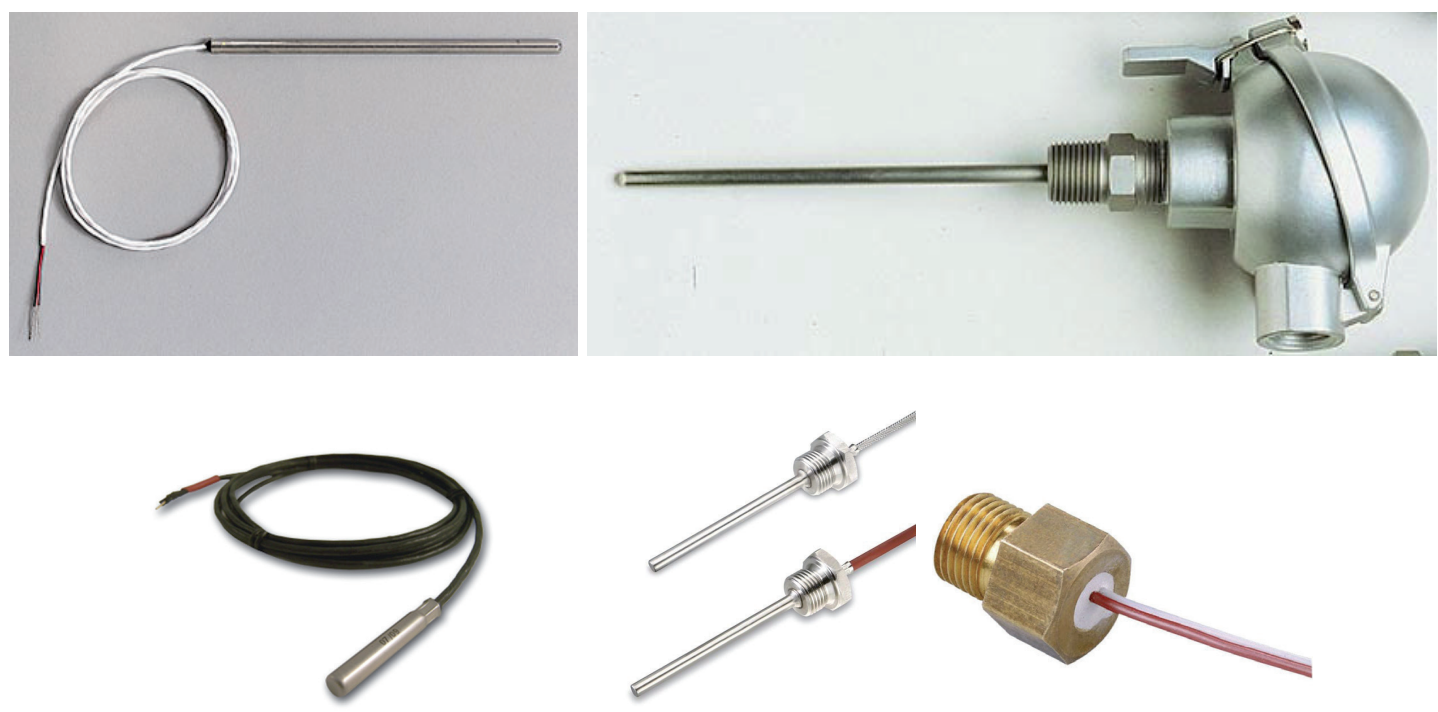

Figure 1: Platinum resistance sensors - various variants for industrial application.

Industrial application includes also environment with chemically aggressive substances with high temperatures and moisture. Sensors for these application are protected with insulation and case with suitable covering of the terminal block. Sensors are applied with thread, screw contact or sticker connection.

From the physic viewpoint, the resistive temperature sensor is as transducer or converter of temperature to other quantities. Dependence of resistive on temperature of the material is used for the measurement. This dependence is known and expressed with table and math model mentioned in standard IEC751 [1]. Marking of this sensors is coming from convention used in practice. For example, Pt100 means that used material is platinum with resistivity $100 \mathrm{ohms}$ at temperature $0^{\circ} \mathrm{C}$. Also it is available Pt1000 sensor which has resistivity $1000 \mathrm{ohms}$ at $0^{\circ} \mathrm{C}$. The standard also provides the math approximation with polynomial function. The standard also includes detailed table about the values of resistivity for relevant temperature value. After measurement of sensor resistivity, you can find the related value of temperature. There are only finite number of values. If your value falls between the values in the table, it is necessary to do linear interpolation to obtain searched value:

\section{$T_{\text {ОКОLIA }}=T_{1}+x$}

Where $T_{1}$ is the nearest value from table in IEC751 related to measured value of resistivity $R_{S} ; x$ ix interpolation temperature increment obtained using the linear interpolation according to data in table from IEC751 [1]

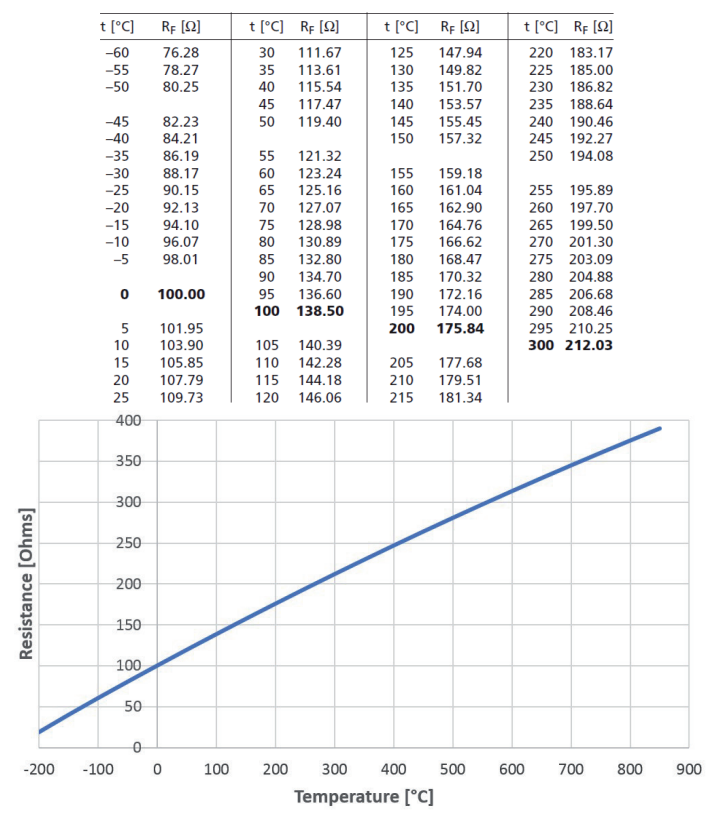

Figure 2: Electrical resistance vs. measured temperature of Pt100 sensor by standard IEC751.

Interpolation temperature increment is a function of

$x=f\left(R_{S}, R_{1}, k_{R T}\right)$

$x=\frac{R_{S}-R_{1}}{k_{R T}}$ 
Where $-k_{R T}$ is transformation coefficient, which is as slope of straight line crossing the points with coordinates of discrete temperature values and related values of electrical resistivity of temperatures sensor. For transformation coefficient is possible to find relationship from function

$k_{R T}=f\left(R_{1}, R_{2}, T_{1}, T_{2}\right)$

$k_{R T}=\frac{R_{2}-R_{1}}{T_{2}-T_{1}}$

$R_{S}$ is electric resistivity of temperature sensor.

$R_{1}, R_{2}$ are the value from table (IEC751) and our measured value of resistivity $R_{S}$ is placed between these values.

$T_{1}, T_{2}$ are value from table, which are related to values of electric resistivity of sensor $R_{1}$ and $R_{2}$.

\section{Estimation of temperature value}

Model of measurement system (Fig. 3) consists of several devices:

- Resistive temperature sensor Pt100 for measurement range $0-600^{\circ} \mathrm{C}$ with maximum accuracy $U_{s}= \pm 0.2 \Omega$.

- Volt meter with data transfer to computer with maximum accuracy $0.000004 \mathrm{~V}$.

- Reference etalon for electric resistivity with nominal value of resistivity $R_{E}=10 \Omega$ with maximum accuracy $U_{\text {RE }}= \pm 0.01 \Omega$.

- Power supply with stabilization of electric current.

Resistive temperature sensor Pt100 is connected in series with resistivity reference etalon. During the measurement, the constant current with values $1 \mathrm{~mA}$ flows through the both these parts (sensor and etalon). Principle of measurement is based on simultaneously measurement of potential drops on both these parts (sensor and etalon). Measurements of voltages are realized with four channel voltmeter connected to computer. For obtaining of the resistivity of temperature sensor Pt100 is possible to use equation:

$R_{S}=\frac{V_{S}}{V_{E}} \cdot R_{E}$

where $V_{S}$ is volt drop on temperature sensor Pt100; $V_{E}$ is volt drop on etalon of electric resistivity; $R_{E}$ is electric resistivity of resistivity etalon; $R_{S}$ is electric resistivity of temperature sensor Pt100.

Equation (3) and (4) create the math model of temperature measurement in working environment using the temperature sensor Pt100. This model shows that it is indirect measurement type.

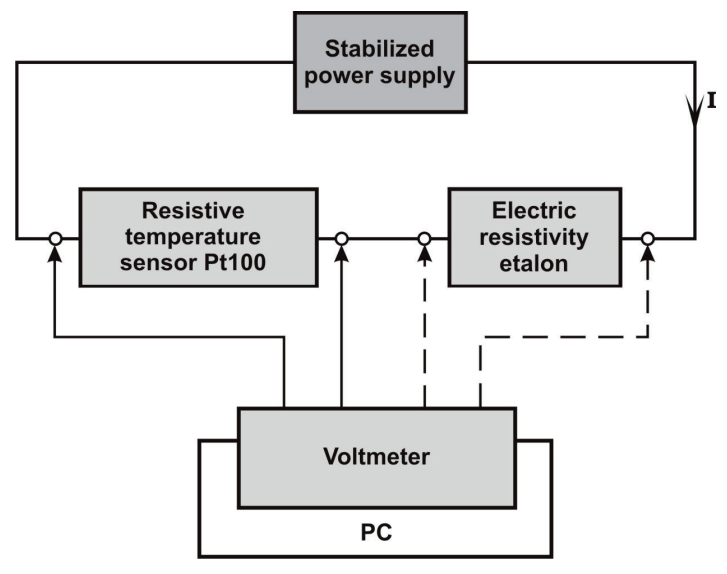

Figure 3: Scheme of the measurement system.

\section{Evaluation of measured data}

Ten measurement of voltage drop have been executed. Measured quantities were voltage drop on electric resistivity sensor and on temperature sensor Pt100. Estimation of average values and standard deviation have been made from obtained measured data. Sources of uncertainties are summarized in the next:

- Standard uncertainty of electric resistivity etalon $u_{B E}$.

- Standard uncertainty of measurement of volt drop on temperature sensor obtained by method A labelled as $u_{\text {AvS }}$.

- Standard uncertainty of measurement of volt drop on temperature sensor obtained by method $B$ labelled as $u_{B v S}$.

- Standard uncertainty of measurement of volt drop on resistive etalon obtained by method $A$ labelled as $u_{A V E}$.

- Standard uncertainty of measurement of volt drop on resistive etalon obtained by method $B$ labelled as $u_{B V E}$.

- Standard uncertainty of transformation coefficient labelled as $u_{\text {BkRT }}$.

- Standard uncertainty of temperature sensor resistivity $R_{1}$ and $R_{2}$ obtained from table included in IEC751 (this is uncertainty obtained by method B).

- Standard uncertainty of temperature $T_{1}$ and $T_{2}$ for sensor related to resistivity $R_{1}$ and $R_{2}$ obtained from table included in IEC751 (this is uncertainty obtained by method $B$ ).

Combined standard uncertainty for measurement of voltage drop can be obtained as square root of quadratic sum of uncertainties obtained by method A and by method B. Standard uncertainty of temperature sensor resistivity results from math model and it is as function of partial uncertainties of all quantities mentioned in math model (4):

$$
u_{R S}=f\left(u_{C V S}, u_{C V E}, u_{B E}\right)
$$


Using the Law of uncertainty propagation, it is possible to write equation for uncertainty of measurement of temperature sensor resistivity:

$u_{R S}=\sqrt{\sum_{i=1}^{n} A_{i}^{2} \cdot u_{i}^{2}}$

Where $A_{i}$ are the sensitivity coefficients of partial quantities mentioned in model (4) and $u_{i}$ are the related uncertainties for these quantities.

Also from model (3) it is possible to obtain the standard uncertainty of transformation coefficient as function of partial uncertainties of all quantities in model (3):

$u_{k_{R T}}=f\left(u_{B R 1}, u_{B R 2}, u_{B T 1}, u_{B T 2}\right)$

Analogically also uncertainty of interpolation temperature increment can be obtained as:

$u_{x}=f\left(u_{R S}, u_{R 1}, u_{k_{R T}}\right)$

Consequently, the final uncertainty for measurement of temperature via using the Pt100 sensor is as function

$u_{T_{O K O L I A}}=f\left(u_{x}, u_{B T 1}\right)$

\section{Conclusions}

The paper brings the proposal for math model of the measurement using the Pt100 resistive temperature sensor with using the table of values mentioned in standard IEC751. This procedure is simplified and there is neglected the covariance between input quantities in math model of measurement (3) and (4). Covariance can deteriorate and also improve the final uncertainty value. It depends on math model and also on values of covariance. Analogically it is possible to do uncertainty balance also for other type of measurement of other quantities in practice [8-16].

\section{Acknowledgments}

\section{The work has been accomplished under the research project APVV- 15-0149, VEGA 1/0224/18, KEGA 006STU-4/2018 financed by the} Slovak Ministry of Education.

\section{References and Notes}

[1] Pt100 Characteristics IEC 751: 1983Industrial Platinum Resistance Thermometer Sensors

[2] Taylor, J. R., An Introduction to Error Analysis: The Study of Uncertainties in Physical Measurements. 1999. University Science Books.
[3] EA-4/02 M:2013 Evaluation of the Uncertainty of Measurement In Calibration. Publication Reference. European Accreditation Laboratory Committee. September 2013 rev 01. cited August, 8th, 2019. Available online: https://european-accreditation.org/wpcontent/uploads/2018/10/ea-4-02-m-rev01-september-2013.pdf.

[4] JCGM 200:2012, International vocabulary of metrology -Basic and general concepts and associated terms(VIM), 3rdEdition (BIPM, 2012).

[5] JCGM 100 - Evaluation of measurement data - Guide to the expression of uncertainty in measurement (ISO/IEC Guide 98-3). First edition September 2008. Available online: http://www.iso.org/sites/ JCGM/GUM-JCGM100.htm; http://www.bipm.org/en/ publications/ guides/gum_print.html.

[6] MSA-L/11 Guidelines on the expressions of uncertainty in quantitative testing (In Slovak) (EA - 4/16: 2003). Guidelines on the expression of uncertainty in quantitative testing. Slovak national accreditation service, SNAS BRATISLAVA, august 2009.

[7] MSA-L/12 Expression of the uncertainty of measurement in calibration (In Slovak) (EA-4/02) - Expression of the uncertainty of measurement in calibration, Slovak national accreditation service, SNAS BRATISLAVA, november 2010.

[8] Palencar, R., Sopkuliak, P., Palencar, J. et al.: Application of Monte Carlo Method for Evaluation of Uncertainties of ITS-90 by Standard Platinum Resistance Thermometer. Measurement Science Review. Volume: 17, Issue: 3 Pages: 108-116 Published: Jun 2017.

[9] Karavaev, Y. L., Kilin, A. A.: Nonholonomic dynamics and control of a spherical robot with an internal omniwheel platform: Theory and experiments, Proceedings of the Steklov Institute of Mathematics, Volume 295, Issue 1, 1 November 2016, Pages 158-167.

[10] Wimmer, G., Palenčár, R., Witkovský, V. (2001). Stochastic models of measurement. (In Slovak) Graphic Studio Ing. Peter Juriga, L. Fullu 13, 84105 Bratislava. 1st. ed., 2001. ISBN 80-968449-2-X.

[11] Chudý, V., Palenčár, R., Kureková, E., Halaj, M. (1999) Measurement of technical quantities (in Slovak). Edition of STU, 1st. ed., 1999. ISBN 80-227-1275-2.

[12] Piňosová, M., Andrejiová, M., Badida, M., Moravec, M., Analysis and Evaluation of Risks from Exposure to Noise in a Working Environment. Acta Mechanica Slovaca 2018, 22(3):44-52 | DOI: 10.21496/ams.2018.026.

[13] Dumek, J., Urban, J., Measurement of Surface Roughness of Volleyball. Acta Mechanica Slovaca 2018, 22(2):34-37 | DOI: 10.21496/ams.2018.015

[14] Diana Šimić Penava, Željko Penava, Željko Knezić, Fabric Thickness Measurement under Tensile Forces. Acta Mechanica Slovaca 2017, 21(4):40-43 | DOI: 10.21496/ams.2017.035.

[15] Kelemen, M.: Model Based Design of Mechatronic Systems. Acta Mechanica Slovaca 2017, 21(4):8-9.

[16] Rónai, L., Szabó, T.: Kinematical investigation and regulation of a 4DOF model robot. Acta Mechanica Slovaca 2016, 20(3):50-56. 